\title{
La neuropathie optique ischémique antérieure bilatérale durant le traitement à l'interféron et à la ribavirine
}

Jason Rinehart OD FAA0; Wilmington VA Medical Center, Wilmington, Delaware

Lisa Stottlemyer OD FAA0; Wilmington VA Medical Center, Wilmington, Delaware

Veuillez adresser toute correspondance à jason.rinehart@va.gov

\begin{abstract}
Résumé
Le virus de l'hépatite $\mathrm{C}(\mathrm{HC})$ est un agent pathogène à transmission hématogène qui cause souvent une infection chronique du foie et expose les patients à la cirrhose, à la décompensation hépatique et au carcinome hépatocellulaire. Le traitement vise à faire disparaître le virus afin de réduire au minimum la morbidité et la mortalité causées par l'hépatite C. Comme traitement traditionnel, on utilise une combinaison interféron pégylé (IFN) et ribavirine qui cause souvent des effets secondaires à la fois systémiques et oculaires. Les hémorragies rétiniennes et les nodules cotonneux constituent les effets secondaires oculaires les plus courants, même si l'on a attribué, dans de rares cas, des événements indésirables ophtalmiques plus importants au traitement à l'IFN.

Nous discutons ici d'un cas de neuropathie optique ischémique antérieure (NOIA) bilatérale qui s'est produit au cours d'un traitement à l'IFN et à la ribavirine contre l'hépatite $C$ de génotype 1 . Nous passons en revue la pathogénèse proposée des effets secondaires oculaires de ce traitement, ainsi que la pathogénèse et les facteurs de risque associés à la NOIA même. Enfin, nous formulerons des recommandations cliniques portant sur le dépistage d'autres problèmes en sus de la rétinopathie si un patient se présente avec des complications ophtalmiques.
\end{abstract}

Mots clés : interféron, hépatite $\mathrm{C}$, neuropathie optique ischémique antérieure, rétinopathie, effets secondaires

\section{Introduction}

Le virus de l'hépatite $\mathrm{C}(\mathrm{VHC})$ est un agent pathogène à transmission hématogène réparti en six grands génotypes qui peut causer des infections tant aiguës que chroniques du foie. De 15 à $25 \%$ des personnes infectées peuvent se débarrasser du virus sans traitement, mais la majorité aura une infection chronique du foie, ce qui les expose au risque de cirrhose, de décompensation hépatique et de carcinome hépatocellulaire ${ }^{1}$. On estime que de 130 à 150 millions de personnes dans le monde vivent avec une infection chronique par le $\mathrm{VHC}^{1,2}$.

Le traitement vise à faire disparaître le virus afin de réduire au minimum la morbidité et la mortalité causées par l'hépatite C. Jusqu'à récemment, le traitement reposait principalement sur une combinaison interféron alpha-pégylé (IFN) et ribavirine administrée pendant 48 semaines. Les effets secondaires du traitement sont essentiellement inévitables et peuvent être très graves. Les progrès rapides des traitements de l'hépatite $\mathrm{C}$ produisent des taux de guérison plus élevés et des effets secondaires moins nombreux que le traitement à l'IFN. Ces nouveaux traitements ne sont toutefois pas encore généralisés pour traiter l'hépatite $C$, principalement parce qu'ils coûtent cher'.

Les effets secondaires ophtalmiques du traitement à l'IFN sont bien connus et entraînent le plus souvent une hémorragie rétinienne et l'apparition de nodules cotonneux qui, souvent, ne sont pas remarqués à cause de l'absence de troubles connexes de la vision. Dans de rares circonstances, on a attribué au traitement à l'IFN des événements indésirables ophtalmiques plus graves, y compris l'occlusion de la veine rétinienne centrale (OVRC) et la neuropathie optique ischémique antérieure (NOIA). Comme ces séquelles oculaires plus inquiétantes obligent à interrompre le traitement afin de laisser la vision s'améliorer, il incomberait à l'optométriste clinique de pouvoir reconnaître ces événements indésirables et leurs liens avec l'utilisation de l'interféron. 


\section{Rapport de cas}

Un homme blanc de 55 ans se présente à la clinique de l'œil se plaignant d'un trouble de la vision qu'il décrit comme une « disparition de points » depuis une semaine. Le patient a des antécédents oculaires sans histoire. Ses antécédents systémiques présentent une hypertension contrôlée par une dose quotidienne de $20 \mathrm{mg}$ d'hydrochlorothiazide/ lisinopril et une hépatite $\mathrm{C}$ chronique de génotype 1 traitée au moyen d'une dose quotidienne de $1200 \mathrm{mg}$ de ribavirine et d'injections hebdomadaires de $180 \mu \mathrm{g}$ d'interféron pégylé alpha $2 \mathrm{~A}$. Le patient avait bien toléré ce traitement et n'avait plus de virus détectable au cours de la $20^{\text {e }}$ semaine de traitement. Au cours de la $22^{\mathrm{e}}$ semaine, une anémie causée par la ribavirine a fait son apparition, accompagnée de symptômes comme l'essoufflement, les étourdissements et la fatigue. Ces symptômes se sont résorbés lorsqu'on a ramené la dose de 1200 à $800 \mathrm{mg}$ par jour. Quatre semaines avant qu'il se présente à la clinique de l'œil, le patient avait une fièvre intermittente avec myalgie, nausées, vomissements et diarrhées. Il a alors été évalué à l'urgence, où il avait une fièvre de $103,7^{\circ} \mathrm{F}$ et une tension artérielle qui était tombée à 99/66.

Lorsqu'il s'est présenté à la clinique de l'œil, le patient en était à la $44^{\mathrm{e}}$ semaine de traitement à l'interféron. Son acuité visuelle était de 20/30 (6/9) dans l'œil droit (OD) et de 20/25 $(6 / 7,5)$ dans l'œil gauche (OS). Les pupilles étaient rondes et réagissaient autant à la lumière sans qu'il y ait de défaut pupillaire afférent. La périmétrie par confrontation était restreinte au niveau supérieur dans chaque œil. L'examen à la lampe à fentes a révélé la présence de cataractes sclérotiques nucléaires résiduelles des deux côtés. Les lectures de tonométrie par applanation de Goldmann s'établissaient à 9 mm Hg OD et $9 \mathrm{~mm} \mathrm{Hg}$ OS à $9 \mathrm{~h} 15$ le matin. La tension artérielle a été mesurée à 112/65 SRA. L'examen du fond d'œil sous pupille dilatée a révélé un œè̀me sectoriel subtil du disque optique associé à des hémorragies en flammèches. Il y avait aussi présence, des deux côtés, d'occlusions en foyer des artérioles rétiniennes (Figure 1). L'examen automatisé du champ visuel a démontré une perte altitudinale supérieure dans chaque œil correspondant aux zones où l'œdème du disque optique était le plus prononcé.

Étant donné la présence atypique d'un œdème de la papille optique des deux côtés, on a soumis le patient à une IRM qui n'a pas révélé d'anomalie intracrânienne ni de stimulation des nerfs optiques. Compte tenu de la gravité de ses symptômes systémiques, le patient a été hospitalisé et a été soumis à un examen de dépistage des maladies infectieuses. On a finalement diagnostiqué chez le patient une rétinopathie associée à l'interféron conjuguée à une neuropathie optique ischémique antérieure non artéritique des deux côtés. On a transmis les résultats au gastroentérologue du patient en lui recommandant d'interrompre le traitement à l'interféron et à la ribavirine.

Le patient a été suivi à la clinique de l'œil pendant six semaines, période au cours de laquelle ses symptômes se sont estompés, son acuité visuelle est revenue à 20/20 (6/6) dans chaque œil et l'œdème du disque optique et les résultats rétiniens se sont résorbés graduellement (Figures 2 et 3 ). On a considéré que les occlusions des artérioles rétiniennes et la neuropathie optique ischémique antérieure pouvaient tous deux contribuer à la perte de champ visuel évocatrice de trouble lorsque le patient s'est présenté. Dans la mesure où la résorption de l'œdème du disque a entraîné une amélioration du défaut du champ visuel, celui-ci était fort probablement une manifestation de NOIA.

Le traitement à l'interféron et à la ribavirine avait obligé à interrompre le traitement et il n'y avait aucun virus détectable au cours de la $20^{\text {e }}$ semaine, situation qui n'a pas changé jusqu'à ce qu'on interrompe le traitement dans sa $44^{e}$ semaine. Après l'interruption du traitement, le patient a eu une rechute et le virus était détectable plusieurs mois plus tard. On a recommencé le traitement à raison de $90 \mathrm{mg}$ de ledipasvir/ $400 \mathrm{mg}$ de sofosbuvir (Harvoni) et de $1200 \mathrm{mg}$ de ribavirine une fois par jour dans les deux cas. Il n'y avait aucun virus détectable 4,8 et 12 semaines après la reprise du traitement.

\section{Discussion}

Les interférons sont des glycoprotéines naturelles qui ont des propriétés antivirales, antitumorales et antiangiogènes ${ }^{4,5}$. Pour le traitement de l'hépatite $\mathrm{C}$, on a administré habituellement l'interféron une fois par semaine sous forme d'injection sous-cutanée. Les patients qui suivent ce traitement ont habituellement de la fièvre, des frissons et des symptômes quasi grippaux. Les nausées, les vomissements et l'hypotension systémique sont possibles, mais rares ${ }^{4,6}$. Les déclarations sur l'incidence de la rétinopathie varient énormément et ces variations sont attribuées à des différences au niveau de la conception de l'étude. L'absence de dépistage officiel entraîne une sous-déclaration tandis que l'évaluation de patients symptomatiques ou à risque entraîne une surdéclaration de séquelles oculaires ${ }^{7}$. Raza et coll. signalent que l'incidence globale de la rétinopathie chez les patients traités à l'interféron et à la ribavirine s'établit à environ $27 \%$. Les patients vivant avec le diabète (DS) et l'hypertension (HTN) sont de 5 à 6 fois plus susceptibles d'avoir une rétinopathie que ceux qui n'ont pas ces problèmes systémiques 5 .

La rétinopathie associée à l'interféron fait habituellement son apparition de 2 à 12 semaines après le début du traitement ${ }^{7}$. Les patients ne présentent en général aucun symptôme visuel, mais ils ont des hémorragies rétiniennes ou des exsudats 
Figure 1

\section{JOUR 1}
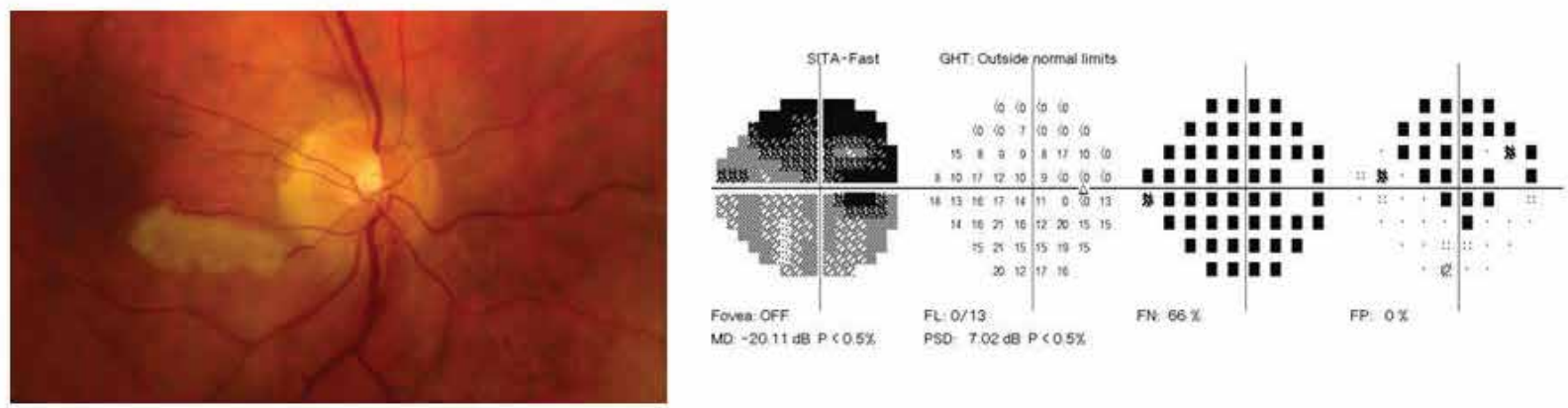

MO $-2011 \mathrm{~dB} P<0.5 \%$

$\mathrm{FL}: 0 / 13$

FN $68 x$

FP $O x$
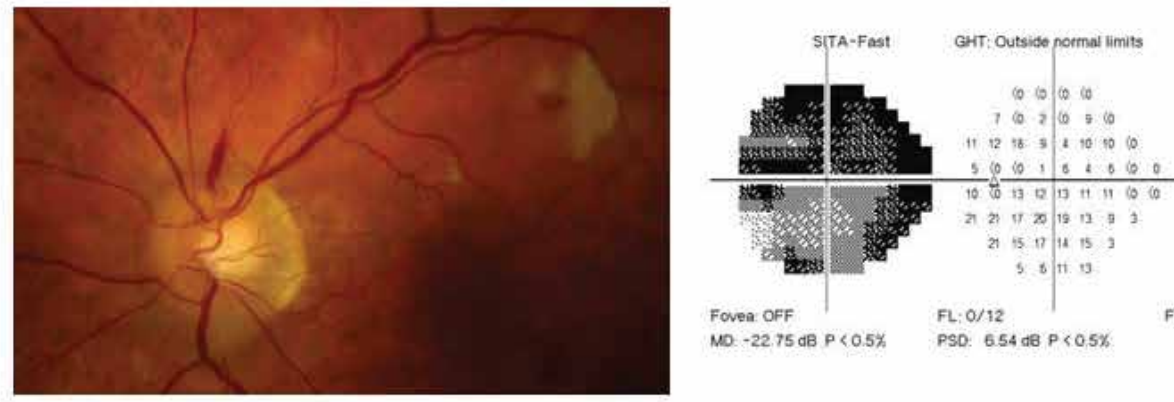

Fovea OFF

MD $-22.75 \mathrm{~dB} P<0.5 \%$

FL: $0 / 12$

PSD. $6.54 \mathrm{de} P<0.5 x$

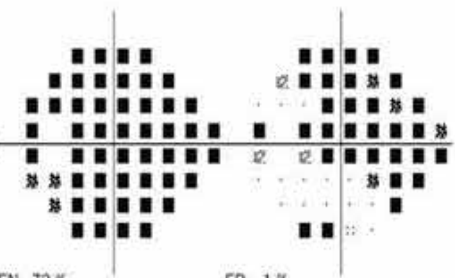

FN $72 x$

FP. $1 x$

\section{JOUR 12}

Figure 2
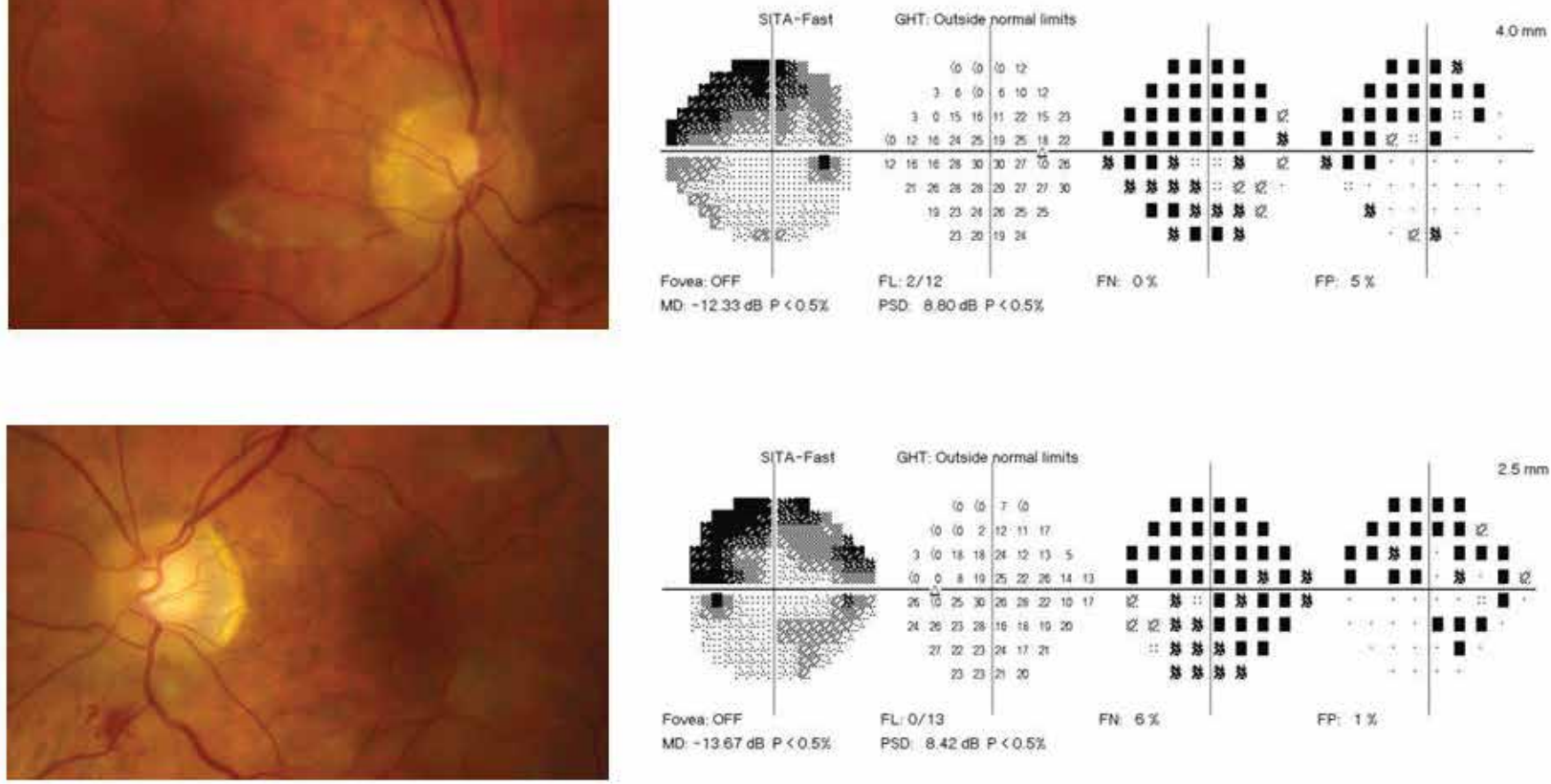

MO $-1367 d B P<0.5 \% \quad P S D \quad 8.42 A B P<0.5 \%$ 
Figure 2

\section{JOUR 45}
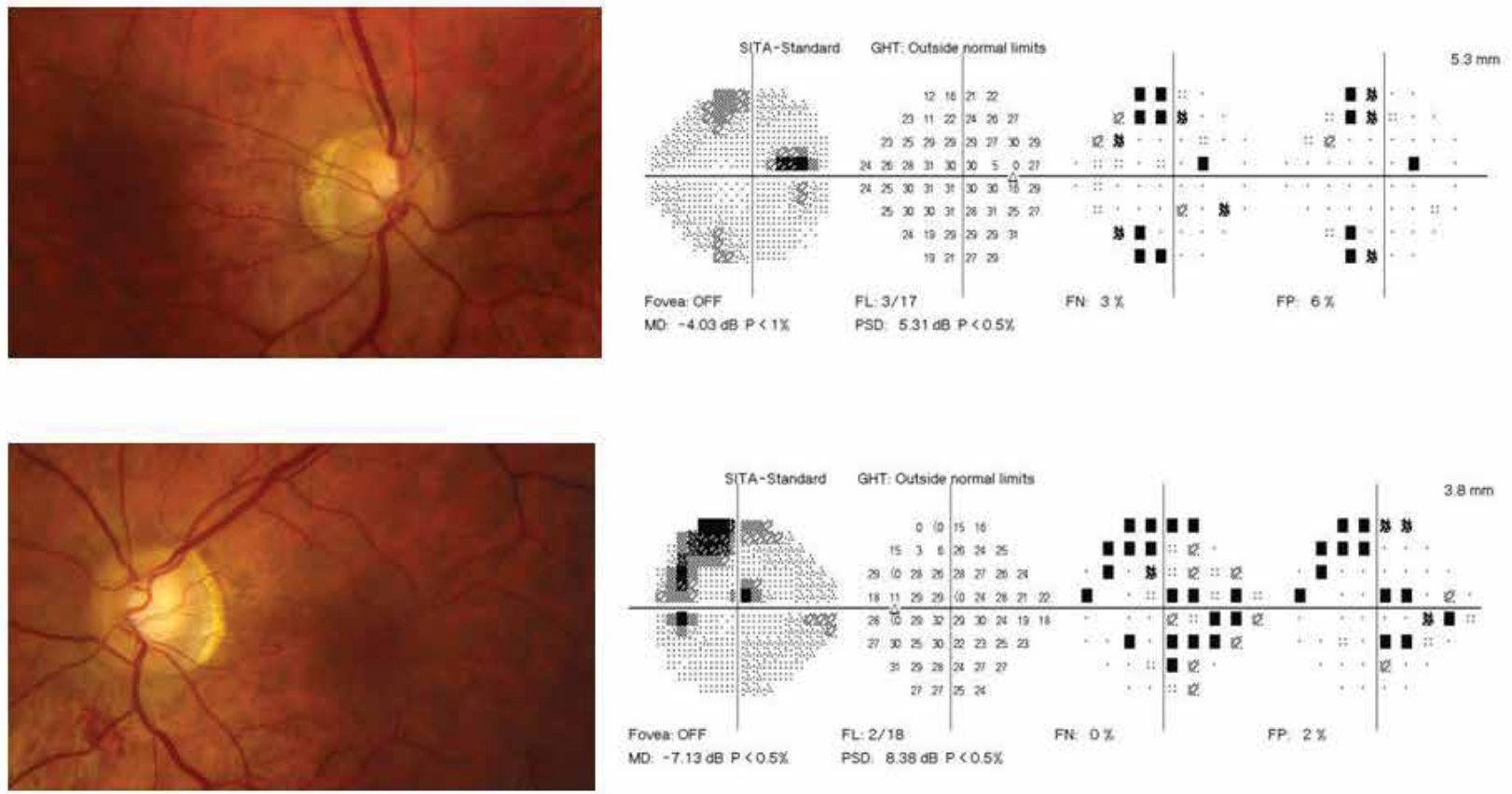

cotonneux révélés par l'examen du fond de l'œil dilaté. On pose en hypothèse que ces changements constituent le résultat final de troubles de la circulation microvasculaire rétinienne ${ }^{8}$. On ne comprend pas à fond la pathophysiologie, mais nombreux sont ceux qui laissent entendre que la dysfonction endothéliale joue un rôle dans le cadre duquel l'agrégation plaquettaire et l'adhérence des leucocytes à l'endothélium vasculaire forment des microcaillots capables de causer des micro-infarctus en foyer $^{7,912}$.

Les interférons agissent en se fixant aux cellules réceptrices et en commençant à produire des protéines effectrices. En cas de NOIA associée à l'interféron, on pose en théorie que ces protéines en circulation pourraient entraîner une hyperviscosité et compromettre la perfusion vers les lits capillaires de la tête du nerf optique ${ }^{6}$. Hayreh soutient depuis longtemps que la NOIA découle d'une diminution transitoire de la perfusion de la papille optique, habituellement au cours du sommeil, et constitue donc un événement hypotensif plutôt qu'un trouble embolique $^{13}{ }^{16}$. L'hypotension systémique est un résultat bien connu associé au traitement à l'interféron. Notre patient a été hospitalisé à cause d'un événement hypotensif au cours des semaines qui ont précédé l'apparition des symptômes visuels.

Il n'existe pas de recommandations normalisées sur le dépistage de la rétinopathie associée à l'interféron chez des patients, probablement parce que la rétinopathie n'est généralement pas associée à la perte de vision, évolue de façon bénigne, est autolimitative et se résorbe habituellement peu après la fin du traitement. En général, la plupart des cliniciens conviennent qu'il est toutefois prudent de soumettre les patients à risque élevé à un dépistage de la rétinopathie liée à l'interféron, mais O'Day et ses collaborateurs sont d'avis que le dépistage de routine est inutile, même dans le cas des patients vivant avec l'hypertension et le diabète sucré, sauf s'ils présentent des symptômes visuels ${ }^{7}$. Comme la perte de vision chez les patients qui ont une rétinopathie diagnostiquée associée à l'interféron est rare, on poursuit en général le traitement même si l'on détermine qu'il y a rétinopathie, étant donné particulièrement le risque plus important pour l'état de santé global des patients chez lesquels on interrompt prématurément le traitement à l'interféron. S'il y a perte de vision au cours du traitement, il faut toutefois soupçonner un événement indésirable moins courant comme une OVRC ou une NOIA. Dans de tels cas, l'interruption du traitement produit le meilleur résultat visuel ${ }^{12}$. 


\section{Dernières réflexions}

Dans le monde toujours mouvant du traitement de l'hépatite $\mathrm{C}$, il semble probable que l'interféron pégylé sera moins présent dans beaucoup de protocoles de traitement, car des agents antiviraux à action directe comme le Sovaldi et l'Harvoni offrent des traitements de plus courte durée, des taux plus élevés de guérison et causent moins d'effets secondaires. Pour un certain nombre de raisons, toutefois, y compris le coût du traitement, l'interféron continuera d'avoir sa place dans l'arsenal thérapeutique du traitement de l'hépatite $\mathrm{C}$ et il devient nécessaire de comprendre les séquelles oculaires possibles associées à ce traitement afin d'optimiser le soin des patients en cause.

\section{Références}

1. Centers for Disease Control and Prevention. Viral Hepatitis - Hepatitis C Information [Internet]. Atlanta, GA : Centres de prévention et de lutte contre la maladie; [mis à jour le 31 mai 2015; cité le 9 juillet 2015]. Disponible à : http://www.cdc.gov/hepatitis/hcv/hcvfaq.htm\#section1

2. Organisation mondiale de la Santé. Centre des médias. Hépatite C - Aidemémoire [Internet]. Genève, Suisse : Organisation mondiale de la Santé; [mis à jour en avril 2014; cité le 9 juillet 2015]. Disponible à : http://www. who.int/mediacentre/factsheets/fs164/fr/

3. Kohli A, Shaffer A, Sherman A, et coll. Treatment of hepatitis C: a systematic review. JAMA. 13 août 2014;312(6):631-40.
4. Fraunfelder FW, Fraunfelder FT. Interferon alfa-associated anterior ischemic optic neuropathy. Ophthalmology. Févr. 2011;118(2):408-11.e1-2.

5. Raza A, Mittal S, Sood GK. Interferon-associated retinopathy during the treatment of chronic hepatitis C: a systematic review. J Viral Hepat. Sept. 2013;20(9):593-9.

6. Purvin VA. Anterior ischemic optic neuropathy secondary to interferon alfa. Arch Ophthalmol. Août 1995;113(8):1041-4.

7. O’Day R, Gillies MC, Ahlenstiel G. Ophthalmologic complications of antiviral therapy in hepatitis $\mathrm{C}$ treatment. World J Gastroenterol. 7 décembre 2013;19(45):8227-37.

8. Okuse C, Yotsuyanagi H, Nagase Y et coll. Risk factors for retinopathy associated with interferon alpha- $2 \mathrm{~b}$ and ribavirin combination therapy in patients with chronic hepatitis C. World J Gastroenterol. 21 juin 2006;12(23):3756-9.

9. Nagaoka T, Sato E, Takahashi A et coll. Retinal circulatory changes associated with interferon-induced retinopathy in patients with hepatitis $\mathrm{C}$. Invest Ophthalmol Vis Sci. Janv. 2007;48(1):368-75.

10. Guyer DR, Tiedeman J, Yannuzzi LA et coll. Interferon-associated retinopathy. Arch Ophthalmol. Mars 1993;111(3):350-6.

11. Nishiwaki H, Ogura Y, Miyamoto K et coll. Interferon alfa induces leukocyte capillary trapping in rat retinal microcirculation. Arch Ophthalmol. Juin 1996;114(6):726-30

12. Selcukbiricik F, Tural D, Senel TE et coll. Bilateral Ischemic Optic Neuropathy Developed under Interferon Therapy. Case Rep Ophthalmol Med. 2012;2012:102739.

13. Hayreh SS. Ischemic optic neuropathies - where are we now? Graefes Arch Clin Exp Ophthalmol. Août 2013;251(8):1873-84.

14. Miller NR. Current concepts in the diagnosis, pathogenesis, and management of nonarteritic anterior ischemic optic neuropathy. J Neuroophthalmol. Juin 2011;31(2):e1-3.

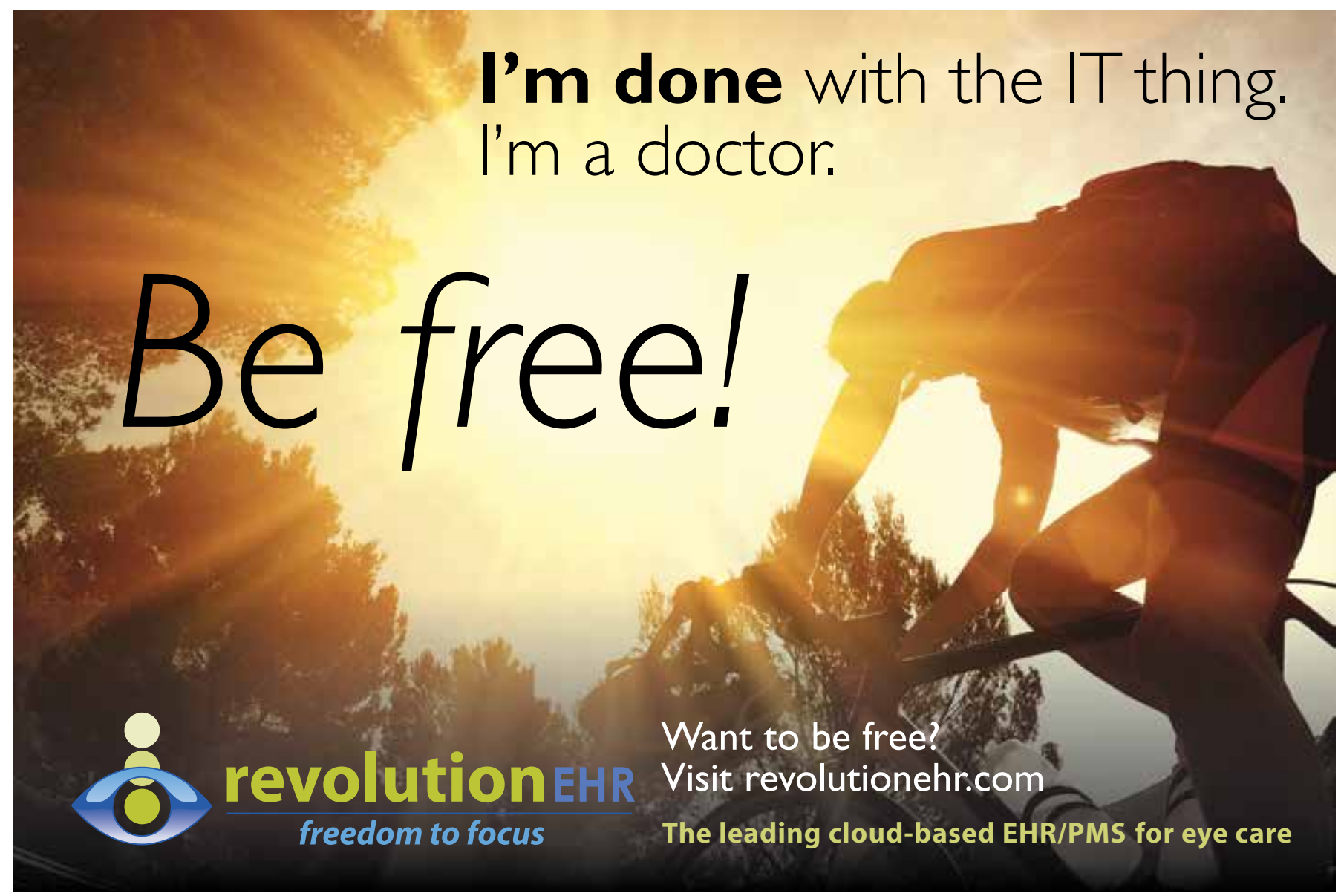

